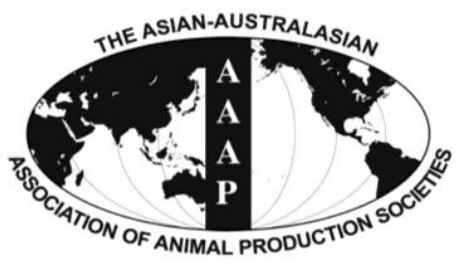

Asian-Aust. J. Anim. Sci.

Vol. 25, No. 11 : 1529-1539 November 2012

http://dx.doi.org/10.5713/ajas.2012.12474

www.ajas.info

pISSN 1011-2367 elSSN 1976-5517

\title{
A Whole Genome Association Study on Meat Quality Traits Using High Density SNP Chips in a Cross between Korean Native Pig and Landrace
}

\author{
K.-T, Lee ${ }^{1, a}$, Y.-M. Lee ${ }^{\text {a }}$, M. Alam ${ }^{\text {a }}$, B. H. Choi ${ }^{1}$, M. R. Park ${ }^{1}$, K.-S. Kim ${ }^{2}$, T.-H. Kim ${ }^{1, a}$ and J.-J. Kim* \\ School of Biotechnology, Yeungnam University, Gyeongsan, Korea
}

\begin{abstract}
A whole genome association (WGA) study was performed to detect significant polymorphisms for meat quality traits in an $\mathrm{F}_{2}$ cross population $(\mathrm{N}=478)$ that were generated with Korean native pig sires and Landrace dams in National Livestock Research Institute, Songwhan, Korea. The animals were genotyped using Illumina porcine 60k SNP beadchips, in which a set of 46,865 SNPs were available for the WGA analyses on ten carcass quality traits; live weight, crude protein, crude lipids, crude ash, water holding capacity, drip loss, shear force, CIE L, CIE a and CIE b. Phenotypes were regressed on additive and dominance effects for each SNP using a simple linear regression model, after adjusting for sex, sire and slaughter stage as fixed effects. With the significant SNPs for each trait $(p<0.001)$, a stepwise regression procedure was applied to determine the best set of SNPs with the additive and/or dominance effects. A total of 106 SNPs, or quantitative trait loci (QTL) were detected, and about 32 to $66 \%$ of the total phenotypic variation was explained by the significant SNPs for each trait. The QTL were identified in most porcine chromosomes (SSCs), in which majority of the QTL were detected in SSCs 1, 2, 12, 13, 14 and 16. Several QTL clusters were identified on SSCs 12, 16 and 17, and a cluster of QTL influencing crude protein, crude lipid, drip loss, shear force, CIE a and CIE b were located between 20 and $29 \mathrm{Mb}$ of SSC12. A pleiotropic QTL for drip loss, CIE L and CIE b was also detected on SSC16. These QTL need to be validated in commercial pig populations for genetic improvement in meat quality via marker-assisted selection. (Key Words: Whole Genome Association, SNP, Meat Quality, Korean Native Pig, Landrace)
\end{abstract}

\section{INTRODUCTION}

Consumers' preference on pork has enabled to produce high meat in pig industry. To meet this ends, studies on genetics to identify genes or chromosomal regions (QTL) that are associated with meat quality have been performed, and lots of QTLs $(6,347)$ for economically important traits have been reported in pig (http://www.animalgenome.org/ QTLdb/).

Experimental crosses of two genetically divergent breeds, such as wild boar vs. European domestic breeds, or Meishan $v s$. western breeds were extensively used in QTL studies (Andersson et al., 1994; Rothschild et al., 1995,

\footnotetext{
* Corresponding Author: Jong-Joo Kim. Tel: +82-53-810-3027, Fax: +82-53-801-3027, E-mail: kimjj@ynu.ac.kr

1 Animal Genomics and Bioinformatics Division, National Institute of Animal Science, Suwon, Korea.

${ }^{2}$ Department of Animal Science, Chungbuk National University, Cheongju, Korea.

a The authors contributed equally to this work.

Submitted Sept. 4, 2012; Accepted Sept. 27, 2012; Revised Sept. 28, 2012
}

2007; Janss et al., 1997; Bidanel and Rothschild, 2002; Hu et al., 2007). After the first report on genome-wide scan and detection of QTL for growth, length of small intestine, and fat deposition in pig (Andersson et al., 1994), many QTL studies using $\mathrm{F}_{2}$ cross populations were performed on growth and fat deposition (Knott et al., 1998; Paszek et al., 1999; Rohrer, 2000; Wada et al., 2000; Bidanel et al., 2001; Malek et al., 2001a; Kim et al., 2005), carcass traits (Andersson-Eklund et al., 1998; Rohrer et al., 1998), and meat quality (de Koning et al., 1999, 2001; Malek et al., 2001b). Recently, several QTL studies on purebreds for meat quality were also reported (Uemoto et al., 2008; Soma et al., 2011).

Korean native pig (KNP) were reported with good meat qualities such as better redness, less cooking loss and shear force than Yorkshire or Landrace breeds (Cho, 2006), and with good amounts of amino acid compositions that were possibly related to flavor and palatability (Hwang et al., 2004). Kim et al. (2007) and Choi et al. (2011) conducted genome-wide scans using microsatellite markers in an $\mathrm{F}_{2}$ cross population between KNP and Landrace to detect 
Mendelian and parent-of-origin QTL for growth, body composition, and meat quality traits.

Recently, with the developments of high throughput genotyping technologies such as next generation sequencing and high density porcine SNP chips, it is possible to genotype large amount of SNPs, e.g. with the porcine $64 \mathrm{k}$ Illumina Infinium assay, which enabled genome-wide association (GWA) study to detect QTL with high mapping resolution up to 1 or $2 \mathrm{Mb}$ confidence region (Fan et al., 2009; Gorbach et al., 2009; Onteru et al., 2009).

In this study, we performed whole genome scans to find significant sets of SNPs that were related to meat quality using the Illumina porcine $64 \mathrm{k}$ SNP chips in the KNP $\times$ Landrace $\mathrm{F}_{2}$ cross population that was used in Kim et al. (2007) and Choi et al. (2011).

\section{MATERIALS AND METHODS}

\section{Animals and phenotypes}

Data were collected from the QTL experimental population that were produced by crossing Korean Native (KNP) boars and Landrace (LN) sows at National Livestock Research Institute (NLRI), Songhwan, Korea. Ten $\mathrm{F}_{1}$ boars were randomly chosen for inter se mating up to six $F_{1}$ sows to produce 38 full-sib $F_{2}$ families resulting in $490 \quad F_{2}$ progenies from the first $(\mathrm{N}=281)$, second $(\mathrm{N}=130)$, and third $(\mathrm{N}=79)$ parities. The raising and management practices of the animals were described in Choy et al. (2002a; 2002b). The $F_{2}$ individuals were slaughtered at the age of 201 to $251 \mathrm{~d}$ with the average of $214.8 \mathrm{~d}$ at the NLRI slaughterhouse. The average final (live) weight before slaughter was $91.6 \mathrm{~kg}$ with a range of 45.2 to $145 \mathrm{~kg}$. Data measurements on ten carcass quality traits (live weight, crude proteins, crude lipids, crude ash, water holding capacity (WHC), drip loss, shear force, CIE L, CIE a, and CIE b, were described in detail in Choi et al. (2011).

\section{Molecular data}

Using the Infinium HD Assay Ultra Protocol (Illumina), all of the $490 \mathrm{~F}_{2}$ animals were genotyped with the porcine 64k SNP beadchips (Illumina Inc., USA). Raw data were visualized and analyzed with the Genome Studio software (Illumina). For subsequent data analysis, a subset of SNP was selected by removing the SNPs in sex chromosomes. Every SNP from the chip data was screened for the availability of GWA tests. Those SNPs on autosomal chromosomes were removed before WGA testing, which met the following three criteria; i) the number of genotype group with one or none (e.g. only AA genotypes and no $\mathrm{AB}$ or $\mathrm{BB}$ ), ii) with a minor allele frequency less than 0.05 , and iii) with the proportion of genotyped individuals less than $90 \%$.

\section{Statistical analysis}

A SAS GLM procedure (SAS version 9.1) was used to preadjust the animal phenotypes before WGA testing. Sex, sire and slaughter stage were fitted as fixed effects for all meat quality traits, except the live weight, for which the effect of slaughter stage was left out of the model. Then, the residuals of each phenotype were regressed on the additive and dominance SNP effect for each SNP under a simple linear regression. In the model, SNP genotypes with AA, $\mathrm{AB}$ and $\mathrm{BB}$ were assigned as 1,0 , and -1 for the additive effect, and 0, 1, 0 for dominance effect, respectively. For significance threshold, $0.1 \%$ point-wise $\mathrm{p}$ value from $F$ distributions was applied for each SNP test.

Among the significant SNPs, the best set of SNP markers for each trait were selected using the stepwise regression procedures (Neter et al., 1990), because some of the significant SNPs would yield redundant information due to linkage disequilibrium (LD) between closely linked SNPs, i.e. a non-random association between alleles of different SNPs. Inclusion and exclusion of each SNP out of the model was determined at $\mathrm{p}<0.001$ level.

With the additive and/or dominance effects of the significant SNPs that were determined by the stepwise regression procedures, variation explained by each $\left(j^{\text {th }}\right)$ SNP $\left(S_{S N P j}^{2}\right)$ was calculated as $\sum_{i=1}^{3} \alpha_{i}^{2} f_{i}-\mu^{2}$, where $i$ denotes each genotype, $\alpha_{i}$ is the estimated additive and/or dominance effects that were included in the multiple SNPs model, e.g. $-\bar{a}, \bar{d}$, and $+\bar{a}$ for $\mathrm{BB}, \mathrm{AB}$, and $\mathrm{AA}$, respectively, for the SNP both with additive $(\bar{a})$ and dominance $(\bar{d})$ effects, $f_{i}$ is the frequency of $i^{\text {th }}$ genotype, $\mu$ is the population mean that can be expressed as $\bar{a}\left(f_{A A}-f_{B B}\right)$ $+\bar{d} f_{A B}$ (Falconer and Mackay, 1996). Proportion of phenotypic variance due to the $j^{\text {th }}$ SNP was then estimated as $S_{S N P j}^{2} / S_{P}^{2}$, in which $S_{P}^{2}$ was phenotype variance that was obtained from residual values of the trait after adjusting fixed and/or covariate effects. The proportion of phenotype variance due to all of the significant SNPs was estimated as $\left(\left(R S S_{n o S N P}-R S S_{S N P S}\right) / R S S_{n o S N P}\right)$, where $R S S$ is residual sum of squares for the model with or without fitting the significant SNPs of each trait.

\section{RESULTS AND DISCUSSION}

Summary statistics for the ten meat quality traits were displayed in Table 1. The coefficient of variation (CV) for crude lipid was the greatest $(96 \%)$, followed by for drip loss (87\%). Among the color scores, CIE b had the greatest CV (43\%), while the lowest CV was observed for crude protein $(5 \%)$.

A set of 46,865 SNPs was chosen from the 62,163 SNPs in the Illumina porcine beadchip (Table 2). The number of 
Table 1. Summary statistics for $490 \mathrm{~F}_{2}$ phenotypes on meat quality in a $\mathrm{KNP} \times \mathrm{LN}$ cross population

\begin{tabular}{|c|c|c|c|c|c|}
\hline Trait & Average & Std. Dev. $^{\mathrm{a}}$ & Minimum & Maximum & $\mathrm{CV}^{\mathrm{b}}$ \\
\hline Live weight (kg) & 91.6 & $15.8(14.0)$ & 45.2 & 145.0 & 17.3 \\
\hline Crude protein $(\%)$ & 22.1 & $1.1(1.0)$ & 16.4 & 25.3 & 5.0 \\
\hline Crude lipid (\%) & 2.1 & $2.0(1.8)$ & 0.1 & 23.6 & 96.2 \\
\hline Crude ash $(\%)$ & 1.0 & $0.1(0.1)$ & 0.8 & 2.9 & 12.5 \\
\hline Water holding capacity $(\%)$ & 57.5 & $5.3(4.8)$ & 45.9 & 80.2 & 9.3 \\
\hline Drip loss (\%) & 2.3 & $2.0(1.8)$ & 0.0 & 11.1 & 86.7 \\
\hline Shear force $(\mathrm{kg})$ & 3.7 & $1.2(1.2)$ & 1.3 & 9.9 & 33.4 \\
\hline CIE L & 50.7 & $4.5(4.0)$ & 34.7 & 67.1 & 8.8 \\
\hline CIE a & 9.7 & $2.5(2.3)$ & 4.5 & 18.3 & 26.0 \\
\hline CIE b & 5.1 & $2.2(1.9)$ & 0.7 & 14.3 & 42.8 \\
\hline
\end{tabular}

${ }^{a}$ Standard deviation. The values in parenthesis indicate residual standard deviations that were obtained for the phenotypes after preadusting for appropriate fixed effects.

${ }^{\mathrm{b}}$ Coefficient of variation $(\%)$

SNPs $(6,622)$ was the greatest for Sus scrofa chromosome (SSC) 1, while SSC12 had the smallest number of SNPs (1,098). The SSCs 4, 7, 13, and 14 had more than 3,000 SNPs. The physical map with all of the available SNPs spanned about 2,424 Mb with an average distance of $44.6 \pm 133.5 \mathrm{~Kb}$ between adjacent SNPs. However, the average distances were different between chromosomes, ranging between $35.6 \mathrm{~Kb}$ in SSC17 and $87.3 \mathrm{~Kb}$ in SSC6. Only $75 \%$ of the SNPs that were embedded in the porcine chip were available in this study, partly due to ascertainment bias, i.e. the Illumina porcine $64 \mathrm{k}$ SNP beadchip was based on the genetics of other commercial breeds, not of Korean native pigs.

A total of 106 SNPs were detected in various chromosomes by the stepwise regression procedures, and the sets of SNPs for the ten carcass quality traits explained significant proportion of phenotypic variance, ranging between $31.8 \%$ for WHC and $65.6 \%$ for drip loss (Table 3 ). For live weight, twelve SNPs (QTL) were found on SSCs 1, $2,5,7,13,14,15$, and 16, of which multiple QTL were located on SSCs 5, 7, 13, and 14 (Table 3). Murani et al. (2005) performed association analyses in Pietrain and Duroc $\times$ Pietrain populations, and found QTL for ham weight on SSC2 (EPOR gene at $52 \mathrm{Mb}$ ), which was closely located

Table 2. The numbers of available SNPs in the Korean native pig and Landrace $\mathrm{F}_{2}$ population and average interval distances between adjacent SNPs in all 18 Sus scrofa autosomes (SSC)

\begin{tabular}{rrrrr}
\hline SSC & Number of SNPs $^{\mathrm{a}}$ & Average interval $(\mathrm{kb})$ & Standard deviation $(\mathrm{kb})$ & Total distance $^{\mathrm{b}}(\mathrm{bp})$ \\
\hline 1 & 6,622 & 44.6 & 133.5 & $295,554,054$ \\
2 & 2,677 & 57.3 & 169.2 & $153,324,765$ \\
3 & 2,178 & 67.1 & 201.1 & $146,204,919$ \\
4 & 3,693 & 39.1 & 90.2 & $144,452,577$ \\
5 & 2,257 & 45.9 & 141.2 & $103,518,423$ \\
6 & 1,967 & 87.3 & 236.3 & $171,673,133$ \\
7 & 3,554 & 37.5 & 34.9 & $133,307,931$ \\
8 & 2,224 & 67.5 & 168.1 & $149,995,098$ \\
9 & 2,638 & 58.5 & 189.8 & $154,365,393$ \\
10 & 1,363 & 57.0 & 182.5 & $77,744,768$ \\
11 & 1,862 & 45.2 & 111.3 & $84,223,424$ \\
12 & 1,098 & 62.2 & 170.1 & $68,252,608$ \\
13 & 3,633 & 59.9 & 168.9 & $217,637,564$ \\
14 & 4,056 & 36.7 & 28.6 & $148,678,088$ \\
15 & 2,659 & 63.7 & 204.8 & $169,455,966$ \\
16 & 1,574 & 55.5 & 168.4 & $87,281,785$ \\
17 & 1,672 & 35.6 & 56.9 & $59,523,835$ \\
18 & 1,138 & 51.8 & 129.2 & $58,905,756$
\end{tabular}

\footnotetext{
${ }^{\mathrm{a}}$ Among the 62,163 SNPs in the Illumina Porcine 64k SNP beadchip, each SNP was selected for whole genome association (WGA) tests if the number of genotype group is two or three, the minor allele frequency was greater than 0.05 , and proportion of genotyped individuals was greater than $90 \%$.

${ }^{\mathrm{b}}$ The distances between the first and the last SNPs that were located on their respective chromosomes.
} 
Table 3. Identities, positions of the SNPs with significant effects on meat quality traits in a Korean native pig and Landrace cross population

\begin{tabular}{|c|c|c|c|c|c|c|c|c|c|}
\hline Trait/SNP marker $^{\mathrm{a}}$ & $\mathrm{SNP}^{\mathrm{b}}$ & SSC & $\mathrm{Kb}^{\mathrm{c}}$ & Additive $^{\mathrm{d}}$ & $\mathrm{SE}^{\mathrm{e}}$ & Dominance $^{f}$ & $\mathrm{SE}^{\mathrm{e}}$ & $-\log _{10} \mathrm{P}^{\mathrm{g}}$ & $\% \sigma_{p}^{2 h}$ \\
\hline Live weight $(\mathrm{kg})$ & & & & & & & & & 36.70 \\
\hline ALGA0002905 & {$[\mathrm{A} / \mathrm{G}]$} & 1 & 35,240 & 1.42 & 0.85 & 4.81 & 1.17 & 2.19 & 3.40 \\
\hline DRGA0003167 & {$[\mathrm{T} / \mathrm{C}]$} & 2 & 47,367 & - & - & 4.47 & 1.15 & 4.00 & 2.52 \\
\hline ALGA0030609 & {$[\mathrm{A} / \mathrm{G}]$} & 5 & 8,998 & 2.28 & 0.83 & - & - & 4.16 & 1.33 \\
\hline ASGA0095684 & {$[\mathrm{A} / \mathrm{G}]$} & 5 & 13,541 & - & - & 5.81 & 1.13 & 4.13 & 4.14 \\
\hline M1GA0010442 & {$[\mathrm{T} / \mathrm{G}]$} & 7 & 62,213 & - & - & 5.15 & 1.12 & 4.14 & 3.38 \\
\hline M1GA0010269 & {$[\mathrm{T} / \mathrm{G}]$} & 7 & 50,229 & -3.39 & 0.84 & - & - & 4.20 & 2.66 \\
\hline INRA0040139 & {$[\mathrm{T} / \mathrm{C}]$} & 13 & 29,753 & - & - & 4.79 & 1.12 & 4.61 & 2.90 \\
\hline CASI0007467 & {$[\mathrm{T} / \mathrm{C}]$} & 13 & 95,955 & - & - & 5.03 & 1.29 & 4.97 & 2.76 \\
\hline INRA0043228 & {$[\mathrm{A} / \mathrm{G}]$} & 14 & 25,540 & 2.51 & 0.97 & 4.46 & 1.36 & 3.96 & 3.79 \\
\hline DRGA0014720 & {$[\mathrm{T} / \mathrm{C}]$} & 14 & 136,395 & -2.79 & 0.81 & 2.59 & 1.20 & 3.30 & 3.02 \\
\hline ASGA0068956 & {$[\mathrm{A} / \mathrm{C}]$} & 15 & 14,116 & -1.39 & 0.77 & 4.26 & 1.23 & 3.44 & 2.69 \\
\hline H3GA0045861 & {$[\mathrm{T} / \mathrm{C}]$} & 16 & 2,073 & - & - & 4.26 & 1.17 & 4.36 & 2.28 \\
\hline Crude protein $(\%)$ & & & & & & & & & 40.90 \\
\hline INRA0000425 & {$[\mathrm{A} / \mathrm{G}]$} & 1 & 6,735 & 0.24 & 0.08 & 0.36 & 0.10 & 3.26 & 5.31 \\
\hline ASGA0006575 & {$[\mathrm{T} / \mathrm{C}]$} & 1 & 221,913 & 0.19 & 0.07 & 0.25 & 0.10 & 4.66 & 2.69 \\
\hline ASGA0009469 & {$[\mathrm{T} / \mathrm{C}]$} & 2 & 8,808 & -0.40 & 0.09 & -0.52 & 0.12 & 2.96 & 9.92 \\
\hline MARC0040826 & {$[\mathrm{T} / \mathrm{C}]$} & 9 & 37,768 & - & - & -0.46 & 0.08 & 5.70 & 4.88 \\
\hline MARC0073944 & {$[\mathrm{A} / \mathrm{G}]$} & 10 & 37,348 & - & - & -0.35 & 0.08 & 3.57 & 2.92 \\
\hline ASGA0054479 & {$[\mathrm{T} / \mathrm{C}]$} & 12 & 20,575 & -0.22 & 0.06 & - & - & 217 & 2.16 \\
\hline H3GA0034945 & {$[\mathrm{T} / \mathrm{C}]$} & 12 & 28,254 & 0.29 & 0.06 & 0.23 & 0.09 & 2.06 & 5.19 \\
\hline ALGA0067249 & {$[\mathrm{A} / \mathrm{C}]$} & 12 & 28,672 & -0.62 & 0.14 & 0.39 & 0.15 & 7.07 & 12.70 \\
\hline MARC0009546 & {$[\mathrm{T} / \mathrm{C}]$} & 12 & 65,388 & 0.46 & 0.06 & - & - & 2.41 & 10.70 \\
\hline DRGA0016110 & {$[\mathrm{A} / \mathrm{G}]$} & 16 & 20,494 & 0.24 & 0.05 & - & - & 4.16 & 2.99 \\
\hline H3GA0046813 & {$[\mathrm{A} / \mathrm{G}]$} & 16 & 36,848 & -0.14 & 0.06 & 0.28 & 0.08 & 2.26 & 2.86 \\
\hline Crude lipid (\%) & & & & & & & & & 57.30 \\
\hline ASGA0098229 & {$[\mathrm{A} / \mathrm{C}]$} & 6 & 31,333 & 0.60 & 0.15 & -0.73 & 0.19 & 5.43 & 6.97 \\
\hline MARC0057020 & {$[\mathrm{T} / \mathrm{C}]$} & 6 & 39,767 & -0.99 & 0.25 & -0.81 & 0.28 & 5.50 & 9.54 \\
\hline H3GA0022262 & {$[\mathrm{A} / \mathrm{G}]$} & 7 & 94,000 & 0.18 & 0.10 & 0.72 & 0.14 & 2.00 & 4.02 \\
\hline DIAS0002084 & {$[\mathrm{T} / \mathrm{C}]$} & 12 & 14,931 & 0.30 & 0.14 & - & - & 3.32 & 0.52 \\
\hline M1GA0016777 & {$[\mathrm{A} / \mathrm{C}]$} & 12 & 21,752 & 0.36 & 0.09 & - & - & 4.06 & 2.12 \\
\hline MARC0004712 & {$[\mathrm{A} / \mathrm{G}]$} & 12 & 27,642 & 0.46 & 0.11 & - & - & 2.90 & 3.18 \\
\hline ALGA0067216 & {$[\mathrm{T} / \mathrm{C}]$} & 12 & 28,517 & -1.27 & 0.26 & 1.03 & 0.32 & 4.03 & 10.30 \\
\hline ALGA0067249 & {$[\mathrm{A} / \mathrm{C}]$} & 12 & 28,672 & 0.55 & 0.24 & -0.72 & 0.26 & 7.07 & 5.23 \\
\hline ASGA0099848 & {$[\mathrm{A} / \mathrm{G}]$} & 12 & 61,692 & -0.44 & 0.12 & -0.48 & 0.14 & 11.04 & 4.42 \\
\hline MARC0037347 & {$[\mathrm{A} / \mathrm{C}]$} & 13 & 19,655 & -1.26 & 0.23 & -1.15 & 0.27 & 3.51 & 15.50 \\
\hline Crude ash (\%) & & & & & & & & & 54.50 \\
\hline ALGA0008433 & {$[\mathrm{A} / \mathrm{G}]$} & 1 & 205,467 & -0.01 & 0.01 & -0.03 & 0.01 & 6.46 & 5.60 \\
\hline DRGA0002289 & {$[\mathrm{A} / \mathrm{G}]$} & 1 & 221,787 & -0.16 & 0.02 & -0.16 & 0.03 & 11.32 & 13.1 \\
\hline ALGA0028117 & {$[\mathrm{A} / \mathrm{G}]$} & 4 & 108,735 & - & - & -0.04 & 0.01 & 2.63 & 3.31 \\
\hline ALGA0037714 & {$[\mathrm{T} / \mathrm{C}]$} & 6 & 43,718 & -0.12 & 0.02 & -0.13 & 0.02 & 8.07 & 8.05 \\
\hline ASGA0035362 & {$[\mathrm{A} / \mathrm{G}]$} & 7 & 100,680 & -0.05 & 0.01 & -0.07 & 0.02 & 2.76 & 4.00 \\
\hline M1GA0015451 & {$[\mathrm{T} / \mathrm{C}]$} & 11 & 62,440 & -0.04 & 0.01 & -0.04 & 0.01 & 4.84 & 6.65 \\
\hline MARC0055183 & {$[\mathrm{T} / \mathrm{G}]$} & 14 & 84,908 & - & - & -0.17 & 0.03 & 6.32 & 6.78 \\
\hline ALGA0093831 & {$[\mathrm{A} / \mathrm{G}]$} & 17 & 20,677 & 0.07 & 0.02 & - & - & 5.71 & 7.21 \\
\hline MARC0115266 & {$[\mathrm{T} / \mathrm{G}]$} & 17 & 25,587 & 0.18 & 0.02 & -0.12 & 0.02 & 10.98 & 10.20 \\
\hline ASGA0076045 & {$[\mathrm{T} / \mathrm{C}]$} & 17 & 25,646 & 0.05 & 0.01 & -0.06 & 0.02 & 6.31 & 8.18 \\
\hline H3GA0048306 & {$[\mathrm{T} / \mathrm{C}]$} & 17 & 25,884 & -0.13 & 0.02 & -0.06 & 0.02 & 5.82 & 6.14 \\
\hline H3GA0050644 & {$[\mathrm{T} / \mathrm{C}]$} & 18 & 14,299 & 0.08 & 0.02 & 0.06 & 0.02 & 2.02 & 1.97 \\
\hline
\end{tabular}


Table 3. Identities, positions of the SNPs with significant effects on meat quality traits in a Korean native pig and Landrace cross population (Continued)

\begin{tabular}{|c|c|c|c|c|c|c|c|c|c|}
\hline Trait/SNP marker $^{\mathrm{a}}$ & $\mathrm{SNP}^{\mathrm{b}}$ & SSC & $\mathrm{Kb}^{\mathrm{c}}$ & Additive $^{\mathrm{d}}$ & $\mathrm{SE}^{\mathrm{e}}$ & Dominance $^{\mathrm{f}}$ & $\mathrm{SE}^{\mathrm{e}}$ & $-\log _{10} \mathrm{P}^{\mathrm{g}}$ & $\% \sigma_{p}^{2 h}$ \\
\hline Water holding capacity (\%) & & & & & & & & & 31.80 \\
\hline DRGA0002791 & {$[\mathrm{A} / \mathrm{G}]$} & 2 & 11,761 & 3.30 & 0.67 & -3.15 & 0.85 & 2.76 & 13.60 \\
\hline ALGA0015239 & {$[\mathrm{T} / \mathrm{C}]$} & 2 & 60,944 & -1.59 & 0.42 & 2.01 & 0.56 & 4.52 & 7.12 \\
\hline H3GA0027388 & {$[\mathrm{T} / \mathrm{C}]$} & 9 & 28,089 & 0.87 & 0.29 & -1.74 & 0.42 & 4.55 & 4.89 \\
\hline MARC0005717 & {$[\mathrm{A} / \mathrm{G}]$} & 9 & 55,154 & 1.00 & 0.33 & 1.34 & 0.45 & 2.06 & 4.04 \\
\hline H3GA0032491 & {$[\mathrm{A} / \mathrm{G}]$} & 11 & 57,624 & 1.05 & 0.36 & - & - & 5.19 & 1.67 \\
\hline ALGA0068125 & {$[\mathrm{T} / \mathrm{C}]$} & 13 & 8,452 & - & - & 3.53 & 0.79 & 2.98 & 3.89 \\
\hline ALGA0107329 & {$[\mathrm{A} / \mathrm{G}]$} & 13 & 10,030 & 1.13 & 0.32 & -1.25 & 0.44 & 2.54 & 4.20 \\
\hline ALGA0069109 & {$[\mathrm{T} / \mathrm{C}]$} & 13 & 17,678 & - & - & -1.64 & 0.40 & 4.80 & 2.88 \\
\hline ASGA0063188 & {$[\mathrm{A} / \mathrm{C}]$} & 14 & 46,404 & 1.32 & 0.29 & - & - & 3.39 & 3.92 \\
\hline ALGA0097847 & {$[\mathrm{A} / \mathrm{G}]$} & 18 & 19,996 & -1.51 & 0.38 & -2.64 & 0.49 & 4.57 & 11.10 \\
\hline Drip loss $(\%)$ & & & & & & & & & 65.60 \\
\hline ALGA0024098 & {$[\mathrm{A} / \mathrm{C}]$} & 4 & 22,246 & -0.30 & 0.15 & 0.82 & 0.19 & 2.12 & 6.48 \\
\hline ALGA0032158 & {$[\mathrm{A} / \mathrm{G}]$} & 5 & 44,959 & -0.56 & 0.13 & - & - & 3.50 & 4.53 \\
\hline SIRI0000798 & {$[\mathrm{T} / \mathrm{C}]$} & 9 & 51,273 & - & - & 0.81 & 0.17 & 5.31 & 4.98 \\
\hline DBMA0000150 & {$[\mathrm{A} / \mathrm{G}]$} & 10 & 62,774 & 0.37 & 0.12 & - & - & 2.89 & 2.28 \\
\hline MARC0034728 & {$[\mathrm{A} / \mathrm{G}]$} & 12 & 28,182 & -0.36 & 0.12 & - & - & 2.71 & 2.02 \\
\hline M1GA0019793 & {$[\mathrm{T} / \mathrm{A}]$} & 14 & 144,961 & 0.61 & 0.16 & 0.80 & 0.23 & 4.08 & 8.30 \\
\hline ALGA0086538 & {$[\mathrm{A} / \mathrm{G}]$} & 15 & 71,074 & 0.16 & 0.11 & - & - & 2.94 & 0.49 \\
\hline ALGA0087545 & {$[\mathrm{A} / \mathrm{G}]$} & 15 & 87,597 & 0.75 & 0.22 & -1.51 & 0.29 & 3.97 & 17.10 \\
\hline ALGA0090076 & {$[\mathrm{T} / \mathrm{G}]$} & 16 & 17,600 & 1.07 & 0.25 & -1.29 & 0.29 & 5.42 & 19.40 \\
\hline Shear force $(\mathrm{kg})$ & & & & & & & & & 33.80 \\
\hline DIAS0000949 & {$[\mathrm{T} / \mathrm{C}]$} & 1 & 50,544 & -0.23 & 0.08 & - & - & 4.53 & 1.97 \\
\hline ASGA0001969 & {$[\mathrm{T} / \mathrm{C}]$} & 1 & 24,745 & -0.45 & 0.13 & -0.69 & 0.16 & 2.98 & 12.40 \\
\hline H3GA0010032 & {$[\mathrm{T} / \mathrm{G}]$} & 3 & 37,157 & - & - & 0.40 & 0.11 & 3.71 & 2.68 \\
\hline ASGA0015463 & {$[\mathrm{A} / \mathrm{C}]$} & 3 & 41,368 & - & - & 0.61 & 0.15 & 2.06 & 3.57 \\
\hline ASGA0090097 & {$[\mathrm{T} / \mathrm{G}]$} & 8 & 33,752 & 0.54 & 0.13 & - & - & 4.11 & 3.25 \\
\hline ALGA0066628 & {$[\mathrm{A} / \mathrm{C}]$} & 12 & 22,606 & - & - & -0.30 & 0.12 & 4.36 & 1.38 \\
\hline ASGA0057366 & {$[\mathrm{A} / \mathrm{G}]$} & 13 & 27,472 & 0.21 & 0.11 & 0.57 & 0.14 & 2.17 & 6.25 \\
\hline ALGA0072961 & {$[\mathrm{T} / \mathrm{G}]$} & 13 & 104,290 & - & - & -0.37 & 0.11 & 2.11 & 2.08 \\
\hline ASGA0068697 & {$[\mathrm{A} / \mathrm{G}]$} & 15 & 9,394 & - & - & 0.71 & 0.15 & 3.24 & 4.34 \\
\hline ASGA0076077 & {$[\mathrm{A} / \mathrm{G}]$} & 17 & 25,853 & -0.56 & 0.18 & -0.82 & 0.24 & 3.44 & 10.00 \\
\hline ALGA0123685 & {$[\mathrm{A} / \mathrm{C}]$} & 18 & 5,709 & -0.22 & 0.07 & -0.37 & 0.11 & 3.50 & 4.24 \\
\hline CIE L & & & & & & & & & 35.60 \\
\hline H3GA0002682 & {$[\mathrm{T} / \mathrm{C}]$} & 1 & 105,840 & - & - & -2.01 & 0.35 & 4.40 & 5.71 \\
\hline INRA0004641 & {$[\mathrm{A} / \mathrm{C}]$} & 1 & 129,084 & 2.83 & 0.66 & 3.25 & 0.75 & 3.74 & 20.50 \\
\hline ASGA0009152 & {$[\mathrm{T} / \mathrm{C}]$} & 2 & 5,830 & -0.43 & 0.24 & 1.36 & 0.33 & 2.31 & 3.34 \\
\hline H3GA0006967 & {$[\mathrm{A} / \mathrm{G}]$} & 2 & 38,822 & - & - & -1.37 & 0.40 & 2.03 & 2.18 \\
\hline ASGA0013908 & {$[\mathrm{A} / \mathrm{C}]$} & 3 & 7,587 & -1.04 & 0.28 & 1.01 & 0.39 & 2.78 & 4.42 \\
\hline ASGA0018311 & {$[\mathrm{A} / \mathrm{C}]$} & 4 & 10,150 & -1.37 & 0.49 & 2.34 & 0.58 & 4.18 & 9.02 \\
\hline ALGA0031253 & {$[\mathrm{A} / \mathrm{G}]$} & 5 & 16,789 & 1.54 & 0.37 & -1.75 & 0.47 & 4.11 & 8.84 \\
\hline CASI0007856 & {$[\mathrm{A} / \mathrm{G}]$} & 11 & 51,107 & 0.58 & 0.32 & 1.60 & 0.40 & 2.28 & 4.47 \\
\hline ASGA0063465 & {$[\mathrm{A} / \mathrm{G}]$} & 14 & 56,703 & 0.92 & 0.23 & 0.88 & 0.33 & 2.36 & 3.81 \\
\hline H3GA0042335 & {$[\mathrm{T} / \mathrm{C}]$} & 14 & 129,502 & - & - & -1.02 & 0.33 & 2.11 & 1.60 \\
\hline ALGA0090076 & {$[\mathrm{T} / \mathrm{G}]$} & 16 & 17,600 & 2.09 & 0.47 & -1.80 & 0.56 & 5.42 & 11.00 \\
\hline ALGA0117025 & {$[\mathrm{A} / \mathrm{G}]$} & 16 & 2,204 & -1.87 & 0.40 & -2.31 & 0.59 & 3.95 & 9.70 \\
\hline
\end{tabular}

to the QTL (47 Mb) in this study. Soma et al. (2011) for live weight in this study (Table 3).

performed genome-wide scans in Duroc and detected QTL Eleven significant QTL for crude proteins were detected, for carcass length and body length at $102 \mathrm{cM}$ and $105 \mathrm{cM}$ among which multiple QTL, i.e. two, four and two QTL of SSC13 that were in close distance to the QTL (96 Mb) were detected on SSCs 1, 12, and 16, respectively (Table 3, 
Table 3. Identities, positions of the SNPs with significant effects on meat quality traits in a Korean native pig and Landrace cross population (Continued)

\begin{tabular}{|c|c|c|c|c|c|c|c|c|c|}
\hline Trait/SNP marker $^{\mathrm{a}}$ & $\mathrm{SNP}^{\mathrm{b}}$ & SSC & $\mathrm{Kb}^{\mathrm{c}}$ & Additive $^{\mathrm{d}}$ & $\mathrm{SE}^{\mathrm{e}}$ & Dominance $^{f}$ & $\mathrm{SE}^{\mathrm{e}}$ & $-\log _{10} \mathrm{P}^{\mathrm{g}}$ & $\% \sigma_{p}^{2 h}$ \\
\hline CIE a & & & & & & & & & 33.40 \\
\hline H3GA0001957 & {$[\mathrm{A} / \mathrm{G}]$} & 1 & 59,441 & -0.51 & 0.13 & - & - & 3.73 & 2.48 \\
\hline DRGA0001537 & {$[\mathrm{T} / \mathrm{C}]$} & 1 & 117,717 & - & - & -0.54 & 0.19 & 2.88 & 1.38 \\
\hline H3GA0006303 & {$[\mathrm{T} / \mathrm{G}]$} & 2 & 12,449 & 0.40 & 0.14 & 0.54 & 0.20 & 2.42 & 2.83 \\
\hline H3GA0013054 & {$[\mathrm{A} / \mathrm{G}]$} & 4 & 73,157 & 0.62 & 0.19 & -0.96 & 0.26 & 2.68 & 6.24 \\
\hline DRGA0007677 & {$[\mathrm{A} / \mathrm{G}]$} & 7 & 60,323 & -1.55 & 0.36 & -0.91 & 0.40 & 2.84 & 14.61 \\
\hline M1GA0017062 & {$[\mathrm{T} / \mathrm{C}]$} & 12 & 27,394 & -1.57 & 0.18 & -0.45 & 0.22 & 2.14 & 20.35 \\
\hline MARC0022924 & {$[\mathrm{A} / \mathrm{G}]$} & 12 & 28,281 & 0.31 & 0.15 & - & - & 4.57 & 0.75 \\
\hline ASGA0072891 & {$[\mathrm{T} / \mathrm{C}]$} & 16 & 17,448 & 0.42 & 0.13 & - & - & 4.49 & 1.64 \\
\hline CIE b & & & & & & & & & 34.70 \\
\hline ALGA0005280 & {$[\mathrm{A} / \mathrm{G}]$} & 1 & 84,761 & 0.51 & 0.11 & -0.58 & 0.16 & 5.33 & 5.83 \\
\hline ASGA0011457 & {$[\mathrm{T} / \mathrm{C}]$} & 2 & 61,858 & -0.37 & 0.12 & -0.45 & 0.16 & 3.45 & 3.25 \\
\hline DIAS0002056 & {$[\mathrm{T} / \mathrm{C}]$} & 4 & 49,947 & 0.54 & 0.15 & -0.68 & 0.20 & 2.54 & 5.96 \\
\hline ALGA0029408 & {$[\mathrm{T} / \mathrm{C}]$} & 4 & 122,404 & -0.23 & 0.12 & 0.50 & 0.16 & 2.53 & 2.43 \\
\hline INRA0019441 & {$[\mathrm{A} / \mathrm{C}]$} & 5 & 43,477 & 1.13 & 0.27 & -0.81 & 0.32 & 4.23 & 10.14 \\
\hline H3GA0016631 & {$[\mathrm{A} / \mathrm{G}]$} & 5 & 49,319 & -0.36 & 0.14 & 0.67 & 0.18 & 2.20 & 4.42 \\
\hline ALGA0110546 & {$[\mathrm{A} / \mathrm{G}]$} & 6 & 149,012 & 0.48 & 0.12 & - & - & 2.55 & 2.75 \\
\hline ASGA0055250 & {$[\mathrm{A} / \mathrm{G}]$} & 12 & 27,918 & -0.51 & 0.12 & -0.40 & 0.16 & 4.09 & 4.53 \\
\hline ALGA0083078 & {$[\mathrm{A} / \mathrm{G}]$} & 14 & 143,515 & 0.30 & 0.13 & - & - & 4.77 & 0.94 \\
\hline ASGA0088659 & {$[\mathrm{T} / \mathrm{C}]$} & 16 & 3,018 & -0.78 & 0.17 & -0.90 & 0.23 & 4.94 & 9.31 \\
\hline ALGA0090076 & {$[\mathrm{T} / \mathrm{G}]$} & 16 & 17,600 & 0.81 & 0.22 & -0.95 & 0.27 & 5.42 & 9.34 \\
\hline
\end{tabular}

${ }^{\mathrm{a}, \mathrm{c}}$ SNP marker annotations and their positions. ${ }^{\mathrm{b}}$ Alternative nucleotides.

${ }^{\mathrm{d}, \mathrm{f}}$ Estimates of additive and dominance effects of the SNP. ${ }^{\mathrm{e}}$ Standard error.

${ }^{g}$ Negative logarithm of base ten for the comparison-wise $p$-value of the test statistic against the null hypothesis of no SNP effect under the one SNP model.

${ }^{\mathrm{h}}$ Proportion of phenotypic variance explained by each SNP or across all of the significant SNPs for the given trait.

Figure 1). Especially the three of the four QTL on SSC12 were clustered between 20 and $29 \mathrm{Mb}$. In our previous linkage mapping results using the same population as in this study, a QTL for crude protein was detected at $0 \mathrm{cM}$ of SSC1 (Choi et al., 2011). In the proximal region (6 Mb), we detected one QTL for the same trait (Table 3).

Ten QTL for crude lipid were detected on SSCs 6, 7, 12, and 13, of which eight QTL were located on SSC6 and SSC12. The detected QTL explained significant proportion $(57 \%)$ of phenotypic variation (Figure 1, Table 3). Stearns et al. (2005) detected a QTL for fat \% at $19 \mathrm{cM}$ of SSC6 in a cross population of Berkshire and Duroc. We identified one QTL for crude lipid at $31 \mathrm{Mb}$ of the same chromosome (Table 3). Sanchez et al. (2007) detected a QTL for fatty acids (C16:1, Palmitoleic) at $40 \mathrm{cM}$ of SSC12 in a Duroc $\times$ Large White cross, which was located distal to the crude lipid QTL at $29 \mathrm{Mb}$ in this study. Munoz et al. (2007) detected five QTL for fatty acid composition in between 1 and $34 \mathrm{cM}$, and three QTL at between 68 and $76 \mathrm{cM}$ of SSC12 in an Iberian and Landrace $\mathrm{F}_{2}$ cross, in which five QTL for crude lipid were detected in this study (Table 3). Clop et al. (2003) also reported two QTL for unsaturated index and linolenic acid at $34 \mathrm{cM}$ of SSC6 and $31 \mathrm{cM}$ of SSC 12, respectively, in an Iberian $\times$ Landrace $F_{2}$ cross population. In the similar regions, multiple QTL for crude lipid were identified in this study (Table 3).

For crude ash, 12 QTLs were detected on eight SSCs, among which the four QTL on SSC17 were clustered at 21 to $26 \mathrm{Mb}$ regions (Table 3). Ovilo et al. (2002) reported QTL for Haematin in an Iberian $\times$ Landrace $F_{2}$ cross population at $109 \mathrm{cM}$ of SSC4 and $87 \mathrm{cM}$ of SSC7, respectively. Haematin is an iron-containing compound which might be related to the crude ash. We detected two QTL for crude ash at $109 \mathrm{Mb}$ and $101 \mathrm{Mb}$ of the respective chromosomes (Table 3).

Ten QTL for WHC were identified on six SSCs, among which multiple QTL were observed on SSCs 2, 9, and 13 (Table 3). Malek et al. (2001b) detected two QTL for the trait on SSC2 and SSC13 in a cross population of Berkshire and Yorkshire. The QTL locations were at $71 \mathrm{cM}$ and $33 \mathrm{cM}$, respectively, that were close to the QTL in this study (Table 3). Murani et al. (2005) detected a drip loss QTL in EPOR gene region (on SSC2, $52 \mathrm{Mb}$ ) in Pietrain, and Duroc $\times$ Pietrain cross population. Usually drip loss and WHC are considered to be highly correlated each other, and we detected one QTL for WHC at $61 \mathrm{Mb}$ of the same chromosome. 


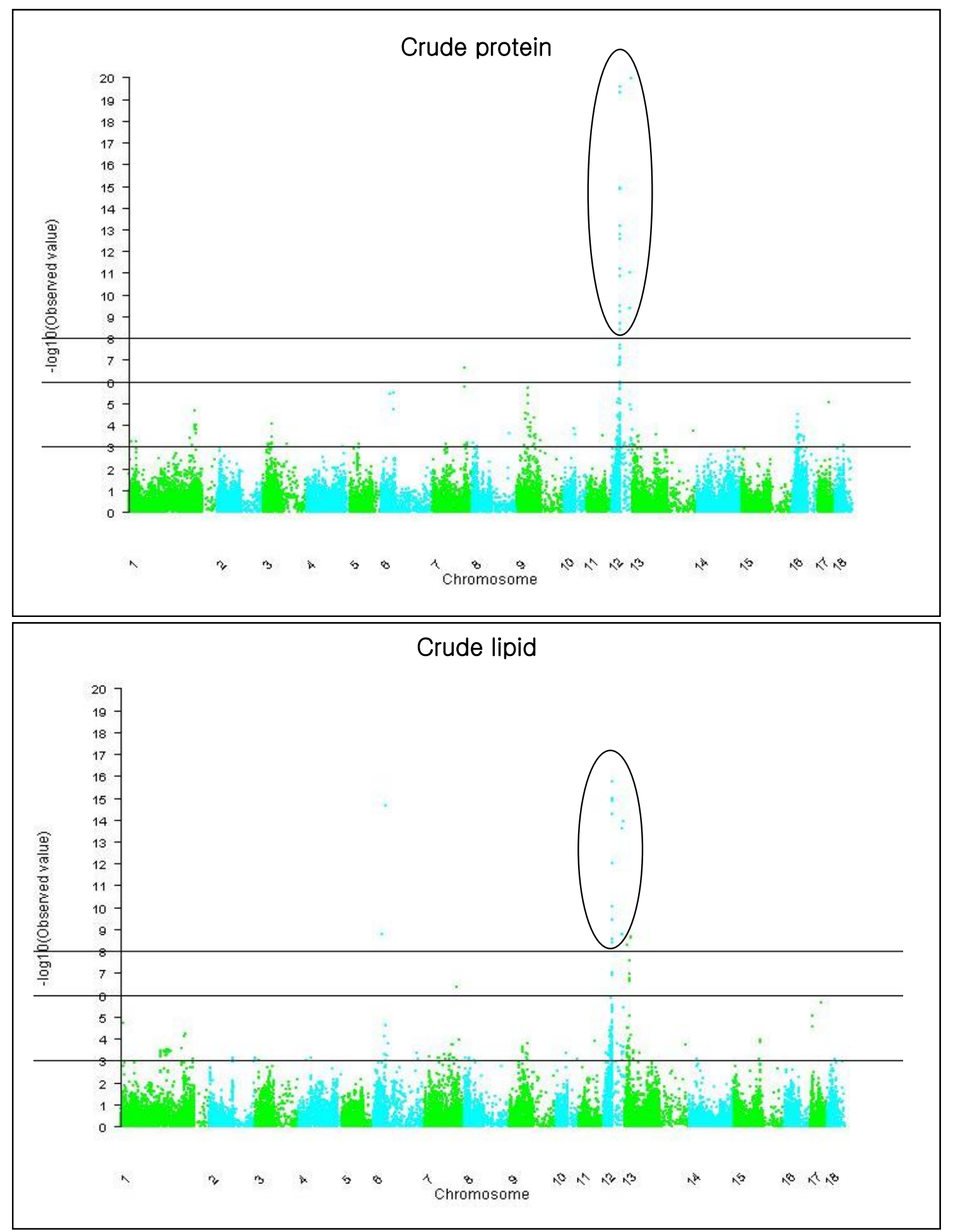

Figure 1. Test statistic profiles for crude protein, crude lipid, crude ash and Cie a under the additive and dominance model for each SNP. Circles indicate highly significant QTL. Y axis indicates $-\log _{10}(\mathrm{p}$-value under the null hypothesis of no SNP effect).

Nine QTL for drip loss were found on SSCs 4, 5, 9, 10, $12,14,15$ and 16, respectively (Table 3 ). Jennen et al. (2007) identified a candidate gene for drip loss, ATF4, at 50 $\mathrm{Mb}$ of SSC5, which was close $(45 \mathrm{Mb})$ to the QTL in this study. Thomsen et al. (2004) found QTL for drip loss on SSC5 and SSC9 in a Berkshire and Yorkshire cross population. In the same chromosomes, two QTL for the trait were detected (Table 3). Li et al. (2011) reported that a SNP in DLX3 gene at $24 \mathrm{Mb}$ of SSC12 was associated with drip loss in the same population as in this study, while a QTL for drip loss was detected at $28 \mathrm{Mb}$ of SSC12 (Table 3). In a Duroc purebred population, Li et al. (2010) found a QTL 


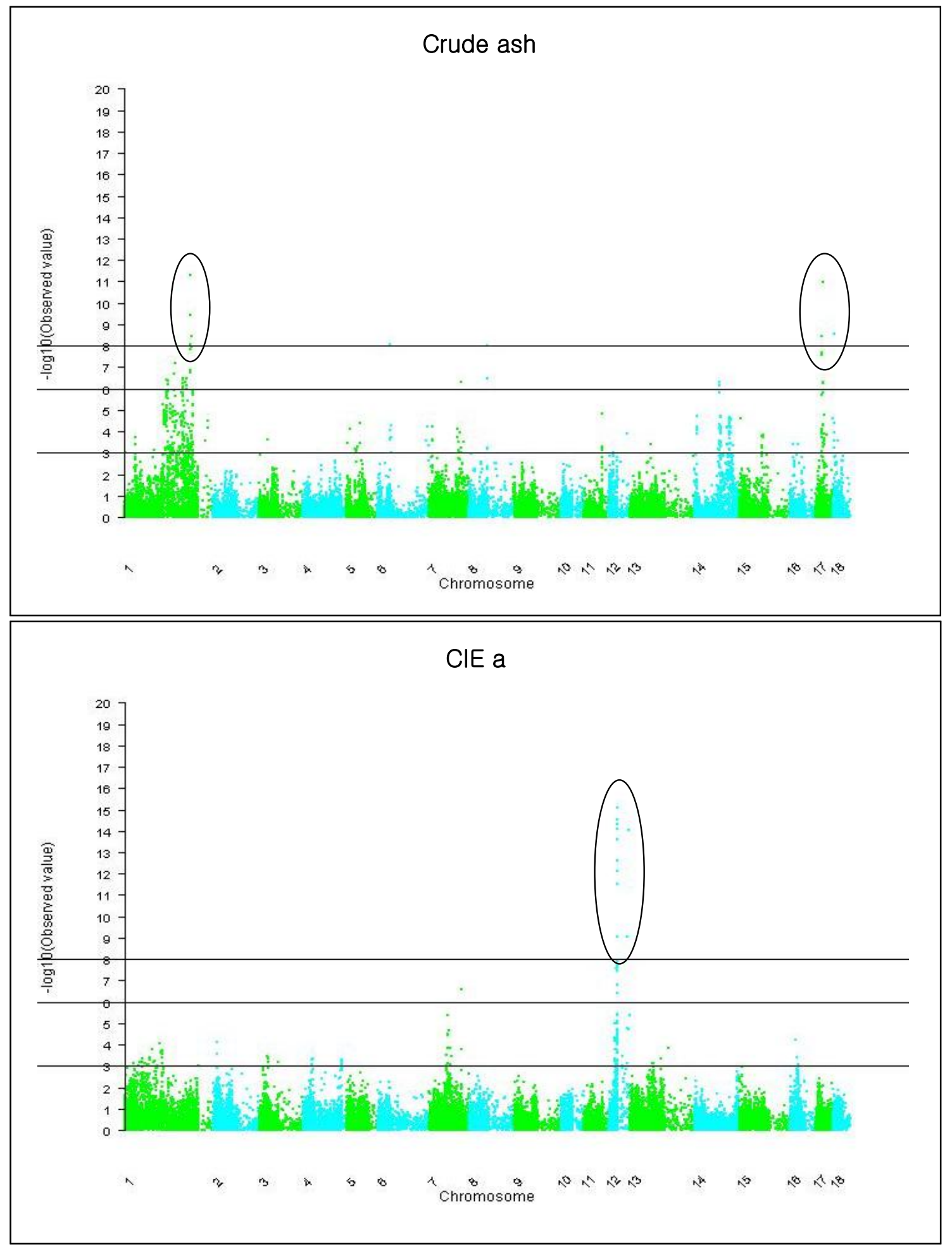

Figure 1. Test statistic profiles for crude protein, crude lipid, crude ash and Cie a under the additive and dominance model for each SNP. Circles indicate highly significant QTL (Continued). Y axis indicates - $\log _{10}$ (p-value under the null hypothesis of no SNP effect).

for the trait on SSC15 (63 cM), and Murani et al. (2005) also reported that the TTN gene (at $79 \mathrm{Mb}$, Ensembl database) on SSC15 was associated with drip loss in a Duroc $\times$ Pietrain cross population. In this study, a QTL for drip loss was detected at $71 \mathrm{Mb}$ (Table 3).
Eleven QTLs for shear force were detected on eight SSCs, of which multiple QTL were located on SSCs 1, 3 and 13 (Table 3). Choi et al. (2011) reported a QTL for shear force at $19 \mathrm{cM}$ of SSC15 that was detected under a line-cross model based linkage analyses using the same 
population as in this study. Thomsen et al. (2004) also detected one QTL for tenderness at $13 \mathrm{cM}$ of the same SSC in a Berkshire $\times$ Yorkshire cross population. In this study, one QTL for shear force was detected at $9 \mathrm{Mb}$ of the same chromosome (Table 3). Murani et al. (2005) reported the PDGFRA gene on SSC8 (35 Mb) in a Duroc cross population, and the NME1 gene on $\operatorname{SSC} 12(25 \mathrm{Mb})$ in Duroc $\times$ Pietrain crosses, which were associated with shear force. Results from Li et al. (2011) and Choi et al. (2011), in which the same $\mathrm{KNP} \times \mathrm{LN}$ cross population was used as in this study, included two QTL for shear force on SSC12 and SSC15, respectively, which were closely located to the two QTL (23 Mb, $9 \mathrm{Mb})$ on the respective SSCs in this study (Table 3).

Most QTL for meat color traits (CIE L, a, b) were found in many chromosomes, especially on SSCs 1, 2, 4, 5, 11, 12, 14, and 16 (Table 3). In some SSC regions, many QTL for meat color were reported; on SSC4 and SSC7 for Minolta a (Ovilo et al., 2002), on SSC2 and SSC14 for Hunter L, and on SSC5 for Hunter b (Roher et al., 2005), on SSC2 for A value (Streans et al., 2005), on SSC4 for color 24hSM (Ma et al., 2009), on SSC1 for L value, on SSC14 for A value, and on SSC4 and SSC5 for B value (Li et al., 2010), and on SSC14 for CIE L (Li et al., 2011). Our study also detected QTL for CIE a, b, and L in these QTL regions (Table 3). Duthie et al. (2008) reported an epistatic effect of OPTOSTAR for CIE L and $b$ that was located on SSC4, which also resided in the similar region to the QTL for CIE L and b in this study (Table 3). The QTL for CIE a that was located at $27 \mathrm{Mb}$ of SSC12 had the greatest statistical evidence of this QTL study (Table 3, Figure 1).

On SSC12, many QTL were clustered between 20 and $29 \mathrm{Mb}$ for crude protein, crude lipid, drip loss, shear force, CIE a and CIE b (Table 3), suggesting that more than one gene reside in the regions. Also, on SSC16 and SSC17, SNPs with pleiotrophic effects were detected, e.g. ALGA0090076 on SSC16 for drip loss, CIE L, and CIE b (Table 3).

Previously, we performed genome-scans to detect QTL for meat quality using the same $\mathrm{KNP} \times \mathrm{LN}$ cross population as in this study (Choi et al., 2011; Li et al., 2011). However, very limited QTL were confirmed in this study, partly because different QTL mapping approaches were applied with the use of different set of markers. In this study, WGA was applied using high density SNP chips, while Choi et al. (2011) performed QTL analysis with limited numbers of microsatellite markers under the interval mapping models that were based on breed-cross designs with Mendelian and non-Mendelian inheritance mode of the QTL. Also, Li et al. (2011) performed association analyses using limited number of SNPs of candidate genes.

\section{CONCLUSION}

Selection for high growth and lean meat has been extensively implemented in pig industry. However, recent genetic improvement has been focused on better meat qualities. In this study, lots of QTL (108 SNPs) were detected for meat quality, providing a set of genetic markers to implement marker-assisted selection (MAS). Several QTL clusters were identified on SSCs 12, 16, and 17, as well as QTL with pleiotrophic effects, e.g. the QTL for drip loss, CIE L and CIE b on SSC16. Characterization of these QTLs is needed to find causal mutations around the QTL, which would enable MAS to be implemented with greater efficiency and accuracy.

\section{ACKNOWLEDGEMENT}

This work was supported by grants from 2-5-13 Agenda research (PJ006707) from the National Institute of Animal Science and a grant (PJ008068, PJ008089) from the Next Generation BioGreen 21 Program, Rural Development Administration, Republic of Korea and the support of "Cooperative Research Program for Agriculture Science \& Technology Development (PJ009103)", the Rural Development Administration, Republic of Korea.

\section{REFERENCES}

Andersson, L., C. S. Haley, H. Ellegren, S. A. Knott, M. Johansson, K. Andersson, L. Andersson-Eklund, I. Edfors-Lilja, M. Fredholm, I. Hansson, J. Hakansson and K. Lundström. 1994. Genetic mapping of quantitative trait loci for growth and fatness in pigs. Science 263:1771-1774.

Andersson-Eklund, L., L. Marklund, K. Lundstrom, C. S. Haley, K. Andersson, I. Hansson, M. Moller and L. Andersson. 1998. Mapping quantitative trait loci for carcass and meat traits in a Wild boar $\times$ Large White intercross. J. Anim. Sci. 76:694-700.

Choi, B. H., Y.-M. Lee, M. Alam, J.-H. Lee, T.-H. Kim, K.-S. Kim and J. -J. Kim. 2011. Detection of Mendelian and parent-oforigin quantitative trait loci for meat quality in a cross between Korean native pig and Landrace. Asian-Aust. J. Anim. Sci. 24:1644-1650.

Bidanel, J. P. and M. Rothschild. 2002. Current status of quantitative trait locus mapping in pigs. Pig News and Information $23: 39 \mathrm{~N}-53 \mathrm{~N}$.

Bidanel, J. P., D. Milan, N. Iannuccelli, Y. Amigues, M. Y. Boscher, F. Bourgeois, C. J. Caritez, J. Gruand, P. L. Roy, H. Lagant, R. Quintanilla, C. Renard, J. Gellin, L. Ollivier and C. Chevalet. 2001. Detection of quantitative trait loci for growth and fatness in pigs. Genet. Sel. Evol. 33:289-309.

Ovilo, C., A. Clop, J. L. Noguera, M. A. Oliver, C. Barragán, C. Rodríguez, L. Silió, M. A. Toro, A. Coll, J. M. Folch, A. Sánchez, D. Babot, L. Varona and M. Pérez-Enciso 2002. Quantitative trait locus mapping for meat quality traits in an Iberian $\times$ Landrace F2 pig population. J. Anim. Sci. 80:28012808 . 
Cho, S.-H. 2006. Characteristics of carcass and meat qualities in Korean native pigs. In symposium of conservation and utilization of Korean native pigs. Korean Livestock Research Institute, p. 33-50.

Choy, Y. H., G. J. Jeon, T. K. Kim, B. H. Choi and H. W. Chung. 2002a. Ear type and coat color on growth performances of crossbred pigs. Asian-Aust. J. Anim. Sci. 15:1178-1181.

Choy, Y. H., G. J. Jeon, T. K. Kim, B. H. Choi, I. C. Cheong, H. K. Lee, K. S. Seo, S. D. Kim, Y. I. Park and H. W. Chung. 2002b. Genetic analyses of carcass characteristics in crossbred pigs: cross between Landrace sows and Korean wild boars. AsianAust. J. Anim. Sci. 15:1080-1084.

Clop, A., C. Ovilo, M. Perez-Enciso, A. Cercos, A. Tomas, A. Fernandez, A. Coll, J. M. Folch, C. Barragan, I. Diaz, M. A. Oliver, L. Varona, L. Silio, A. Sanchez and J. L. Noguera. 2003. Detection of QTL affecting fatty acid composition in the pig. Mamm. Genome. 14:650-656.

De Koning, D. J., L. L. G. Janss, A. P. Rattink, P. A. van Oers, B. J. de Vries, M. A. M. Groenen, J. J. der Poel, P. N. de Groot, E. W. Brascamp and J. A. M. van Arendonk. 1999. Detection of quantitative trait loci for back fat thickness and intramuscular fat content in pigs (Sus scrofa). Genetics 152:1679-1690.

De Koning, D., B. Harlizius, A. P. Rattink, M. A. M. Groenen, E. W. Brascamp and J. A. M. van Arendonk. 2001. Detection and characterization of quantitative trait loci for meat quality traits in pigs. J. Anim. Sci. 79:2812-2819.

Duthie, C., G. Simm, A. Doeschl-Wilson, E. Kalm, P. W. Knap and R. Roehe. 2008. Quantitative trait loci for chemical body composition traits in pigs and their positional associations with body tissues, growth and feed intake. Anim. Genet. 39:130-140.

Fan, B., S. K. Onteru, D. Garrick, K. J. Stalder and M. F. Rothschild. 2009. A genome-wide association study for pig production and feet and leg structure traits using the PorcineSNP60 BeadChip. Pig Genome III Conference, November 2-4, 2009, Hinxton, Cambridge, UK. Abstract No. 6.

Gorbach, D. M., W. J. Cai, C. M. Dekkers, J. M. Young and D. J. Garrick. 2009. Whole-genome analyses for genes associated with residual feed intake and related traits utilizing the PorcineSNP60 BeadChip. Pig Genome III Conference. November 2-4. Hinxton, UK. Abstract No. 11.

Hu, Z. L., E. R. Fritz and J. M. Reecy. 2007. AnimalQTLdb: A livestock QTL database tool set for positional QTL information mining and beyond. Nucleic Acids Res. 35:D604D609.

Hwang, I. H., B. Y. Park, S. H. Cho, J. H. Kim and J. M. Lee. 2004. Identification of muscle proteins related to objective meat quality in Korean native black pig. Asian-Aust. J. Anim. Sci. 17:1599-1607.

Janss, L. L. G., A. M. Johan, J. A. M. van Arendonk and E. W. Brascamp. 1997. Segregation analyses for presence of major genes affecting growth, backfat, and litter size in Dutch Meishan crossbreds. J. Anim. Sci. 75:2864-2876.

Jennen, D. G. J., A. D. Brings, G. Liu, H. Jüngst, E. Tholen, E. Jonas, D. Tesfaye, K. Schellander and C. Phatsara. 2007. Genetic aspects concerning drip loss and water-holding capacity of porcine meat. J. Anim. Breed. Genet. 124(Suppl. 1):2-11.

Kim, E. H., B. H. Choi, K. S. Kim, C. K. Lee, B. W. Cho, T. H. Kim and J. J. Kim. 2007. Detection of Mendelian and parent- of-origin quantitative trait loci in a cross between Korean native pig and Landrace I. growth and body composition traits. Asian-Aust. J. Anim. Sci. 20:669-676.

Kim, T. H., K. S. Kim, B. H. Choi, D. H. Yoon, G. W. Jang, K. T. Lee, H. Y. Chung, H. Y. Lee, H. S. Park and J. W. Lee. 2005. Genetic structure of pig breeds from Korea and China using microsatellite loci analysis. J. Anim. Sci. 83:2255-2263.

Knott, S. A., L. Marklund, C. S. Haley, K. Andersson, W. Davies, H. Ellegren, M. Fredholm, I. Hansson, B. Hoyheim, K. Lundstrom, M. Moller and L. Andersson. 1998. Multiple markers mapping of quantitative trait loci in a cross between outbred wild boar and large white pigs. Genetics 149:10691080.

Li, H. D., M. S. Lund, O. F. Christensen, V. R. Gregersen, P. Henckel and C. Bendixen. 2010. Quantitative trait loci analysis of swine meat quality traits. J. Anim. Sci. 88:2904-2912.

Li, X., S. -W. Kim, K. -T. Do, Y. -K. Ha, Y. -M. Lee, S. -H. Yoon, H. -B. Kim, J. -J. Kim, B. -H. Choi and K. -S. Kim. 2011. Analyses of porcine public SNPs in coding-gene regions by resequencing and phenotypic association studies. Mol. Biol. Rep. 38:3805-3820.

Liu, G., D. G. J. Jennen, E. Tholen, H. Juengst, T. Kleinwächter, M. Hölker, D. Tesfaye, G. Ün, H. -J. Schreinemachers, E. Murani, S. Ponsuksili, J.-J. Kim, K. Schellander and K. Wimmers. 2007. A genome scan reveals QTL for growth, fatness, leanness and meat quality in a Duroc-Pietrain resource population. Anim. Genet. 38:241-252.

Ma, J., J. Ren, Y. Guo, Y. Duan, N. Ding, L. Zhou, L. Li, X. Yan, K. Yang, L. Huang, Y. Song, J. Xie, D. Milan and L. Huang. 2009. Genome-wide identification of quantitative trait loci for carcass composition and meat quality in a large-scale White Duroc $\times$ Chinese Erhualian resource population. Anim. Genet. 40:637-647.

Malek, M., J. C. M. Dekkers, H. K. Lee, T. Baas and M. F. Rothschild. 2001a. A molecular genome scan analysis to identify chromosomal regions influencing economic traits in the pig. I. Growth and body composition. Mamm. Genome 12: 630-636.

Malek, M., J. C. M. Dekkers, H. K. Lee, T. J. Baas, K. Prusa, E. Huff-Lonergan and M. F. Rothschild. 2001b. A molecular genome scan analysis to identify chromosomal regions influencing economic traits in the pig. II. Meat and muscle composition. Mamm. Genome 12:637-645.

Markljung, E., M. H. Braunschweig, P. Karlskov-Mortensen, C. S. Bruun, M. Sawera, I.-C. Cho, I. Hedebro- Velander, ̊. Josell, K. Lundström, G. von Seth, C. B. Jørgensen, M. Fredholm and L. Andersson. 2008. Genome-wide identification of quantitative trait loci in a cross between Hampshire and Landrace II: Meat quality traits. BMC Genet. 9:22

Munoz, G., E. Alves, A. Fernandez, C. Ovilo, C. Barragan, J. Estelle, R. Quintanilla, J. M. Folch, L. Silio, M. C. Rodriguez and A. I. Fernandez. 2007. QTL detection on porcine chromosome 12 for fatty-acid composition and association analyses of the fatty acid synthase, gastric inhibitory polypeptide and acetyl-coenzyme A carboxylase alpha genes. Anim. Genet. 38:639-646.

Murani, E., M. F. W. te Pas, K. C. Chang, R. Davoli, J. W. M. Merks, H. Henne, R. Wörner, H. Eping, S. Ponsuksili, K. Schellander, N. da Costa, D. Prins, B. Harlizius, E. Knol, M. 
Cagnazzo, S. Braglia and K. Wimmers. 2005. Analysis of effects of genes differentially expressed during myogenesis on pork quality. 56th Annual Meeting of the European Association for Animal Production. June 5-8, Uppsala, Sweden (Abstr)

Neter, J., W. Wasserman and M. H. Kutner. 1990. Applied linear statistical models. $3^{\text {rd }}$ ed. Irwin. Boston.

Onteru, S. K., B. Fan, D. Garrick, K. J. Stalder and M. F. Rothschild. 2009. Whole genome analyses for pig reproductive traits using the PorcineSNP60 BeadChip. Pig Genome III Conference. November 2-4, 2009, Hinxton, Cambridge, UK. Abstract No. 5.

Paszek, A. A., P. J. Wilkie, G. H. Flickinger, G. A. Rohrer, L. J. Alexander, C. W. Beattie and L. B. Schook. 1999. Interval mapping of growth in divergent swine cross. Mamm. Genome 10:117-122.

Ramos, A. M., J. W. M. Bastiaansen, G. S. Plastow and M. F. Rothschild. 2009. Genes located on a SSC17 meat quality QTL region are associated with growth in outbred pig populations. Anim. Genet. 40:774-778.

Rohrer, G. A. 2000. Identification of quantitative trait loci affecting birth characters and accumulation of back fat and weight in Meishan-White Composit resource population. J. Anim. Sci. 78:2547-2553.

Rohrer, G. A. and J. Keele. 1998. Identification of quantitative trait loci affecting carcass composition in swine II. Muscling and wholesale product trait yield traits. J. Anim. Sci. 76:2255-2262.

Rothschild, M. F., H. C. Liu, C. K. Tuggle, T. P. Yu and L. Wang. 1995. Analysis of chromosome 7 genetic markers for growth and carcass performance traits. J. Anim. Breed. Genet. 112: 341-348.

Rothschild, M. F., Z. Hu and Z. Jiang. 2007. Advances in QTL mapping in pigs. Int. J. Biol. Sci. 3:192-197.
Sanchez, M. -P., N. Iannuccelli, B. Basso, J.- P. Bidanel, Y. Billon, G. Gandemer, H. Gilbert, C. Larzul1, C. Legault, J. Riquet, D. Milan and P. L. Roy. 2007. Identification of QTL with effects on intramuscular fat content and fatty acid composition in a Duroc $\times$ Large White cross. BMC Genet. 8:55.

Soma, Y., Y. Uemoto, S. Sato, T. Shibata, H. Kadowaki, E. Kobayashi and K. Suzuki. 2011. Genome-wide mapping and identification of new quantitative trait loci affecting meat production, meat quality, and carcass traits within a Duroc purebred population. J. Anim. Sci. 89:601-608.

Stearns, T. M., J. E. Beever, B. R. Southey, M. Ellis, F. K. McKeith and S. L. Rodriguez-Zas. 2005. Evaluation of approaches to detect quantitative trait loci for growth, carcass, and meat quality on swine chromosomes $2,6,13$, and 18 . I. Univariate outbred F2 and sib-pair analyses. J. Anim. Sci. 83:1481-1493.

Thomsen, H., H. K. Lee, M. F. Rothschild, M. Malek and J. C. M. Dekkers. 2004. Characterization of quantitative trait loci for growth and meat quality in a cross between commercial breeds of swine. J. Anim. Sci. 82:2213-2228.

Uemoto, Y., Y. Nagamine, E. Kobayashi, S. Sato, T. Tayama, Y. Suda, T. Shibata and K. Suzuki. 2008. Quantitative trait loci analysis on Sus scrofa chromosome 7 for meat production, meat quality, and carcass traits within a Duroc purebred population. J. Anim. Sci. 86:2833-2839.

Van Wijk, H. J., B. Dibbits, E. E. Baron, A. D. Brings, B. Harlizius, M. A. M. Groenen, E. F. Knol and H. Bovenhuis. 2006. Identification of quantitative trait loci for carcass composition and pork quality traits in a commercial finishing cross. J. Anim. Sci. 84:789-799.

Wada, Y., T. Akita, T. Awata, T. Furukawa, N. Sugai, Y. Inage, K. Ishii, Y. Ito, E. Kobayashi, H. Kusumoto, T. Matsumoto, S. Mikawa, M. Miyake, A. Murase, S. Shimanuki, T. Sugiyama, Y. Uchida, S. Yanai and H. Yasue. 2000. Quantitative trait loci (QTL) analysis in a Meishan $\times$ Gottingen cross population. Anim. Genet. 31:376-384. 